\title{
Host status of selected cultivated fruit crops to Meloidogyne enterolobii
}

\author{
Vânia M. Freitas • Joelma G. P. Silva • Cesar B. Gomes • \\ José M. C. Castro • Valdir R. Correa • \\ Regina M. D. G. Carneiro
}

Accepted: 7 November 2016/Published online: 21 November 2016

C) Koninklijke Nederlandse Planteziektenkundige Vereniging 2016

\begin{abstract}
Meloidogyne enterolobii (syn. M. mayaguensis) has been reported to cause severe damage in commercial guava orchards and other plants in Central and South American countries. Considering the risk of introduction and dissemination of this pest in the European region, $M$. enterolobii was placed on the EPPO A2 list in 2010. The use of non-host fruit species is a recommended strategy to manage rootknot nematodes in infested guava orchards. This study screened 89 plant genotypes from 25 fruit plants of economic importance, plus two susceptible controls (guava and tomato) for its host status to M. enterolobii. Three to eight months after inoculation, nematode reproduction factor (RF) was used to characterize host suitability of fruit crops to this nematode. Ten banana genotypes, six Barbados cherries, one fig, two grape rootstocks and six
\end{abstract}

V. M. Freitas · J. G. P. Silva • R. M. D. G. Carneiro ( $₫)$

Embrapa Recursos Genéticos e Biotecnologia, CP.02372, Brasília, DF 70849-970, Brazil

e-mail: regina.carneiro@embrapa.br

C. B. Gomes

Embrapa Clima Temperado, Laboratório de Fitopatologia, C.P. 403, Pelotas, RS 96001-970, Brazil

J. M. C. Castro

Embrapa Semi-Árido Tropical, BR 428, Km 152, Petrolina, PE 56302-970, Brazil

V. R. Correa

Instituto Federal de Educação, Ciência e Tecnologia do

Tocantins-Campus Dianópolis, Rodovia TO 040 Km 349,

Dianópolis, TO, Brazil melons were rated as good hosts for this nematode. Sixteen fruit plants behaved either as non-hosts or poor hosts to $M$. enterolobii, including assaí, atemoya, avocado, cashew nut, citrus, coconut, grape, jabuticaba, mango, mulberry, papaya, passion fruit, sapodilla, soursop, starfruit and strawberry. For the future, field experiments in areas infested by this nematode are essential to confirm the greenhouse results. These non-host fruit species can replace in the future eradicated guava trees in fields severely infested by this nematode and become an economic option for growers where $M$. enterolobii is considered a serious problem.

Keywords Crop rotation · Fruit crops · Guava root-knot nematode $\cdot$ Psidium guajava $\cdot$ Resistance

\section{Introduction}

The root-knot nematode (RKN) Meloidogyne enterolobii (syn. M. mayaguensis) has been detected worldwide from a wide range of hosts and causes severe damage to commercial guava orchards (Brito et al. 2007; Carneiro et al. 2012). In Brazil, Cuba, Puerto Rico, Mexico, and Venezuela guava production has declined during the past quarter century due to increasing pressure from root-knot nematodes (El Borai and Duncan 2005). Meloidogyne enterolobii was detected in two commercial greenhouses in Switzerland on tomato (Solanum lycopersicum cv. Maxifort) rootstock resistant to Meloidogyne spp. (Kiewnick et al. 2008). Considering 
the risk of introduction and dissemination of this pest in the European region, $M$. enterolobii was added to the EPPO Alert List (EPPO 2008).

In Brazil, M. enterolobii was originally detected on guava orchards in 2001, in Pernambuco and Bahia states. Since then, this nematode has been a major concern in the country due to its rapid spread, making guava cultivation unviable in heavily infested areas (Carneiro et al. 2001; Carneiro et al. 2012).

Guava has been grown by small farmers in the São Francisco Valley, a major guava-producing region in the northeast of Brazil, resulting in high return on investments and an option for the local labor force. In this region, there are reports of $70 \%$ reduction in guava production in areas infested with $M$. enterolobii, reported within a seven-year period (Carneiro et al. 2007). According to Pereira et al. (2009), direct losses associated with guava infection by $M$. enterolobii in several states in Brazil might reach up to US\$ 61 million. Taken together, these data reveal the importance of this nematode, for which alternative management strategies should be considered in order to reduce damage as well as economic losses.

Considering the difficulty in identifying $M$. enterolobii by just the perineal pattern or host races, it is possible that $M$. enterolobii from guava has been misidentified in several countries, because of its morphological resemblance to $M$. incognita and $M$. arenaria and considering that the identification of root-knot nematodes in this plant species has been based only on morphology (e.g. the perineal patterns) and differential host tests (Carneiro et al. 2001; Carneiro et al. 2012). Meloidogyne enterolobii is considered a polyphagous species, parasitizing not only guava, but also ornamental plants, fruit trees and annual crops (Brito et al. 2007), as well as some wild guavas (Freitas et al. 2014). A list of resistant, immune and susceptible cover crops was presented by Carneiro et al. (2012); however, the polyphagous status of M. enterolobii was not confirmed for those plants.

Moreover, little is known about the host status of alternative fruit crops regarding $M$. enterolobii. It is important to point out to growers alternative crops to be planted in infested areas where guava orchards have been eradicated. Thus, the objective of this study was to determine the host status of 89 plant genotypes from 20 cultivated fruit crops against $M$. enterolobii under greenhouse conditions and provide a list of non-hosts and poor host fruits to this nematode species to be used in future field trials to confirm the results obtained under greenhouse conditions.

\section{Materials and methods}

Plant genotypes

A total of 89 plant genotypes distributed in 20 fruit crop species (scientific names mentioned before) of economic importance, plus tomato cv. Santa Clara and guava (Psidium guajava cv. Paluma) as susceptible controls were used in this study (Tables 1, 2, 3, 4, 5, 6, 7 and 8). Plants were either obtained from seeds (assaí, atemoya, cashew, coconut, mango, melon, papaya, sapodilla, soursop and starfruit) or from cuttings (avocado, banana, Barbados cherry, citrus, fig, grape, jabuticaba, mulberry, passion fruit and strawberry). Plants were grown in 5-L plastic bags filled with a mixture of autoclaved clay-soil, sand and Plantimax compost (1:1:1) under greenhouse conditions at $25-30{ }^{\circ} \mathrm{C}$ and were watered and fertilized as required.

Nematode inoculation

A highly aggressive population of $M$. enterolobii originally collected in Petrolina, (Carneiro et al. 2007) (Pernambuco state) and disseminated in different states in Brazil using guava seedlings (Siqueira et al. 2009) was used in this study due to its pathogenicity on commercial guava. Nematode identification was carried out using esterase (Est) phenotype (Carneiro and Almeida 2001) and SCAR marker as previously described (Tigano et al. 2010). Prior to nematode inoculation, the population was multiplied on tomato plants cv. Santa Clara for three months under greenhouse conditions. Eggs were extracted from infected roots using $0.5 \%$ $\mathrm{NaOCl}$, according to Hussey and Barker (1973), using a blender instead of manual agitation.

Eleven nematode inoculation bioassays were carried out in four greenhouses from 2010 to 2011 (Tables 1,3, 5 and 7) and repeated from 2012 to 2013 (Tables 2, 4, 6 and 8). Seedlings of about $10-15 \mathrm{~cm}$ were inoculated with 10,000 eggs of $M$. enterolobii by pipetting the nematode suspension around the stem base. Plants were arranged in a completely randomized design with eight replicates for each bioassay ( 89 plant genotypes +11 controls were used for all 11 bioassays). Plants were maintained in the greenhouse as mentioned above. 
Table 1 Host status of fruit crops to Meloidogyne enterolobii under controlled conditions three and four months after nematode inoculation carried out in 2010-2011

\begin{tabular}{|c|c|c|c|c|c|c|}
\hline Fruit crops and genotypes & FRW $(g)^{1}$ & $\mathrm{GI}^{2}$ & $\mathrm{EMI}^{2}$ & $\mathrm{NE} / \mathrm{g}_{\text {roots }}{ }^{3}$ & $\mathrm{RF}^{4}$ & Reaction 5 \\
\hline \multicolumn{7}{|l|}{ Melon (Cucumis melo L.) } \\
\hline Espanhol (yellow or common) & 51.63 & 5 & 4.38 & 1662 & $8.58 \mathrm{a}$ & $\mathrm{GH}$ \\
\hline 'Orange lisa' & 20.06 & 5 & 4.50 & 3354 & $6.73 \mathrm{a}$ & $\mathrm{GH}$ \\
\hline 'Japonês' & 24.64 & 4.86 & 4.14 & 2638 & $6.50 \mathrm{a}$ & $\mathrm{GH}$ \\
\hline 'Caipira' & 16.81 & 5 & 3.75 & 2255 & $5.44 \mathrm{a}$ & GH \\
\hline 'Orange verde' & 20.38 & 5 & 4.63 & 2649 & $5.40 \mathrm{a}$ & $\mathrm{GH}$ \\
\hline 'Cantaloupe' & 25.06 & 5 & 4.13 & 1041 & $2.61 \mathrm{~b}$ & $\mathrm{GH}$ \\
\hline Tomato 'Santa Clara' (Solanum lycopersicum L.) - susceptible control & 39 & 5 & 5 & 3469 & $13.53 \mathrm{a}$ & $\mathrm{GH}$ \\
\hline \multicolumn{7}{|l|}{ Strawberry (Fragaria $x$ ananassa Duch) } \\
\hline 'Aromas' & 13.50 & 0 & 0 & 0 & $0 \mathrm{~b}$ & $\mathrm{NH}$ \\
\hline ‘Camarosi’ & 13.24 & 0 & 0 & 0 & $0 \mathrm{~b}$ & $\mathrm{NH}$ \\
\hline 'Camino Real' & 11.13 & 0 & 0 & 0 & $0 \mathrm{~b}$ & $\mathrm{NH}$ \\
\hline 'Diamante' & 12.51 & 0 & 0 & 0 & $0 \mathrm{~b}$ & $\mathrm{NH}$ \\
\hline 'Oso Grande’ & 14.06 & 0 & 0 & 0 & $0 \mathrm{~b}$ & $\mathrm{NH}$ \\
\hline 'Santa Clara' & 8.86 & 0 & 0 & 0 & $0 \mathrm{~b}$ & $\mathrm{NH}$ \\
\hline 'Ventana' & 14.93 & 0 & 0 & 0 & $0 \mathrm{~b}$ & $\mathrm{NH}$ \\
\hline Tomato 'Santa Clara' (Solanum lycopersicum L.) - susceptible control & 42 & 5 & 5 & 3547 & $14.9 \mathrm{a}$ & $\mathrm{GH}$ \\
\hline \multicolumn{7}{|l|}{ Barbados cherry (Malpighia spp.) } \\
\hline $\mathrm{P} 18 \mathrm{~A} 2 \mathrm{~B} 1$ & 6.57 & 4 & 3.90 & 5814 & $3.82 \mathrm{~b}$ & $\mathrm{GH}$ \\
\hline $\mathrm{P} 24 \mathrm{~A} 2 \mathrm{~B} 1$ & 5.53 & 3.80 & 3.10 & 4394 & $2.43 \mathrm{~b}$ & $\mathrm{GH}$ \\
\hline P27A3B5 & 8.77 & 4.50 & 3.90 & 4949 & $4.34 \mathrm{~b}$ & $\mathrm{GH}$ \\
\hline P29A3B5 & 5.09 & 2.70 & 2.70 & 4126 & $2.10 \mathrm{~b}$ & $\mathrm{GH}$ \\
\hline P33A2 & 6.25 & 3.90 & 3.50 & 3008 & $1.88 \mathrm{~b}$ & $\mathrm{GH}$ \\
\hline P34A3B5 & 5.07 & 4.1 & 4.1 & 8757 & $4.44 \mathrm{~b}$ & $\mathrm{GH}$ \\
\hline Tomato 'Santa Clara' (Solanum lycopersicum L.) - susceptible control & 28 & 5 & 5 & 4571 & $12.80 \mathrm{a}$ & $\mathrm{GH}$ \\
\hline
\end{tabular}

Data were transformed as $\log (\mathrm{x}+1)$. Means followed by different letters in the column are significantly different according to Scott-Knot's test $(P<0.05)$. Coefficient of variation $(\mathrm{CV})=25 \%, 28 \%$ and $32 \%$

${ }^{1}$ Mean values $(n=8)$ of fresh root weight (FRW)

${ }^{2}$ Mean values $(n=8)$ of gall index and egg mass index, based on a $0-5$ scale, where 0 : no gall/egg mass, $1: 1-2$ galls/egg mass, 2: 3-10 galls/ egg mass, 3: 11-30 galls/egg mass, 4: 31-100 galls/egg mass and 5: > 100 galls /egg mass (Hartman \& Sasser 1985)

${ }^{3}$ Mean values $(n=8)$ of number of eggs per gram of roots

${ }^{4}$ Reproduction factor $(\mathrm{RF}=$ final population/10,000 eggs of $M$. enterolobii)

${ }^{5} \mathrm{R}=$ reaction of inoculated plants: $\mathrm{RF}<0.1=$ non-host $(\mathrm{NH}), 0.1<\mathrm{RF}<1=$ poor host $(\mathrm{PH})$ and $\mathrm{RF} \geq 1=\operatorname{good}$ host $(\mathrm{GH})($ Sasser et al. 1984)

Three to eight months after inoculation, plants were evaluated for their host status for M. enterolobii. These periods after inoculations included three months (melon and strawberry), four months (Barbados cherry), six months (assaí, banana, cashew, fig, jabuticaba, mango, mulberry, papaya) and eight months (atemoya, avocado, citrus, coconut, grape, passion fruit, sapodilla, soursop, starfruit). The root system was rinsed with tap water and weighed. Roots were stained with phloxine B and evaluated for gall and egg mass numbers (galling index, GI; egg mass index, EMI), using a $0-5$ scale, where $0=$ no galls or egg masses; $1=1-2 ; 2=3-10 ; 3=11-$ $30 ; 4=31-100$; and $5=>100$ galls or egg masses per root system (Hartman and Sasser 1985). The GI and EMI were used only to describe root damage and not to quantify nematode reproduction. Eggs were extracted using a modified extraction method according to Hussey and Barker (1973), using a blender instead of manual 
Table 2 Host status of fruit crops to Meloidogyne enterolobii under controlled conditions after three (melon) and four months (other crops) after nematode inoculation, carried out in 2012 and 2013

\begin{tabular}{|c|c|c|c|c|c|c|}
\hline Fruit crops and genotypes & FRW $(g)^{1}$ & $\mathrm{GI}^{2}$ & $\mathrm{EMI}^{2}$ & $\mathrm{NE} / \mathrm{g}_{\text {roots }}{ }^{3}$ & $\mathrm{RF}^{4}$ & Reaction $^{5}$ \\
\hline \multicolumn{7}{|l|}{ Melon (Cucumis melo L.) } \\
\hline Espanhol (yellow or common) & 32.24 & 5 & 4.25 & 2224 & $7.17 \mathrm{~b}$ & GH \\
\hline 'Orange lisa' & 28.33 & 5 & 4.70 & 2944 & $8.34 \mathrm{~b}$ & $\mathrm{GH}$ \\
\hline 'Japonês' & 25.24 & 5 & 4.38 & 2694 & $6.80 \mathrm{~b}$ & $\mathrm{GH}$ \\
\hline 'Caipira' & 26.32 & 5 & 4.55 & 3245 & $8.54 \mathrm{~b}$ & GH \\
\hline 'Orange verde' & 18.28 & 5 & 4.92 & 4015 & $7.34 \mathrm{~b}$ & $\mathrm{GH}$ \\
\hline 'Cantaloupe' & 25.43 & 5 & 4.54 & 3664 & $9.32 \mathrm{~b}$ & $\mathrm{GH}$ \\
\hline Tomato 'Santa Clara' (Solanum lycopersicum L.) - susceptible control & 42.23 & 5 & 5 & 3746 & $15.82 \mathrm{a}$ & $\mathrm{GH}$ \\
\hline \multicolumn{7}{|l|}{ Strawberry (Fragaria $x$ ananassa Duch) } \\
\hline 'Aromas' & 11.40 & 0 & 0 & 0 & $0 \mathrm{~b}$ & NH \\
\hline ‘Camarosi’ & 12.34 & 0 & 0 & 0 & $0 \mathrm{~b}$ & $\mathrm{NH}$ \\
\hline 'Camino Real' & 15.26 & 0 & 0 & 0 & $0 \mathrm{~b}$ & NH \\
\hline 'Diamante' & 13.14 & 0 & 0 & 0 & $0 \mathrm{~b}$ & $\mathrm{NH}$ \\
\hline ‘Oso Grande’ & 12.80 & 0 & 0 & 0 & $0 \mathrm{~b}$ & $\mathrm{NH}$ \\
\hline 'Santa Clara' & 10.24 & 0 & 0 & 0 & $0 \mathrm{~b}$ & NH \\
\hline 'Ventana' & 11.33 & 0 & 0 & 0 & $0 \mathrm{~b}$ & $\mathrm{NH}$ \\
\hline Tomato 'Santa Clara' (Solanum lycopersicum L.) - susceptible control & 35.20 & 5 & 5 & 5341 & $18.8 \mathrm{a}$ & $\mathrm{GH}$ \\
\hline \multicolumn{7}{|l|}{ Barbados cherry (Malpighia spp.) } \\
\hline P18A2B1 & 10.34 & 5 & 5 & 6712 & $6.94 \mathrm{~b}$ & $\mathrm{GH}$ \\
\hline $\mathrm{P} 24 \mathrm{~A} 2 \mathrm{~B} 1$ & 8.23 & 5 & 5 & 6476 & $5.33 \mathrm{~b}$ & GH \\
\hline P27A3B5 & 7.37 & 5 & 5 & 10,081 & $7.43 \mathrm{~b}$ & $\mathrm{GH}$ \\
\hline P29A3B5 & 9.18 & 5 & 5 & 8932 & $8.20 \mathrm{~b}$ & $\mathrm{GH}$ \\
\hline P33A2 & 7.55 & 5 & 5 & 8185 & $6.18 \mathrm{~b}$ & $\mathrm{GH}$ \\
\hline P34A3B5 & 8.20 & 5 & 5 & 6512 & $5.34 \mathrm{~b}$ & $\mathrm{GH}$ \\
\hline Tomato 'Santa Clara' (Solanum lycopersicum L.) - susceptible control & 32.20 & 5 & 5 & 6149 & $19.80 \mathrm{a}$ & $\mathrm{GH}$ \\
\hline
\end{tabular}

Data were transformed as $\log (\mathrm{x}+1)$. Means followed by different letters in the column are significantly different according to Scott-Knot's test $(P<0.05)$. Coefficient of variation $(\mathrm{CV})=22 \%, 26 \%$ and $27 \%$

${ }^{1}$ Mean values $(n=8)$ of fresh root weight (FRW)

${ }^{2}$ Mean values $(n=8)$ of gall index and egg mass index, based on a $0-5$ scale, where 0 : no gall/egg mass, $1: 1-2$ galls/egg mass, $2: 3-10$ galls/ egg mass, 3: 11-30 galls/egg mass, 4: 31-100 galls/egg mass and 5: > 100 galls /egg mass (Hartman \& Sasser 1985)

${ }^{3}$ Mean values $(n=8)$ of number of eggs per gram of roots

${ }^{4}$ Reproduction factor $(\mathrm{RF}=$ final population/10,000 eggs of $M$. enterolobii)

${ }^{5} \mathrm{R}=$ reaction of inoculated plants: $\mathrm{RF}<0.1=$ non-host $(\mathrm{NH}), 0.1<\mathrm{RF}<1=$ poor host $(\mathrm{PH})$ and $\mathrm{RF} \geq 1=\operatorname{good}$ host $(\mathrm{GH})($ Sasser et al., 1984)

agitation and $1 \% \mathrm{NaOCl}$. Total egg number per plant was quantified under a light microscope using Peters' slides. The reproduction factor $(\mathrm{RF})$, calculated as $\mathrm{RF}=P f \mid P i$, where $P f=$ final nematode population and $P i=$ initial nematode population $(P i=10,000)$, was used to designate host suitability for $M$. enterolobii. The average RF was transformed as $\log 10(x+1)$, submitted to analysis of variance and the means separated using the Scott-Knot test at 5\% confidence level. Plant species and genotypes were classified as follows: $\mathrm{RF}<0.1-$ non-host, $0.1<\mathrm{RF}<1$ - poor host and RF $\geq 1-$ good host (Sasser et al., 1984).

\section{Results}

The bioassays performed in 2010-2011 (Tables 1, 3, 5 and 7) showed a similar host response as the biossays 
Table 3 Host status of fruit crops to Meloidogyne enterolobii under controlled conditions six months after nematode inoculation carried out in 2010 and 2011

\begin{tabular}{|c|c|c|c|c|c|c|}
\hline Fruit crops and genotypes & FRW $(g)^{1}$ & $\mathrm{GI}^{2}$ & $\mathrm{EMI}^{2}$ & $\mathrm{NE} / \mathrm{g}_{\text {roots }}{ }^{3}$ & $\mathrm{RF}^{4}$ & Reaction ${ }^{5}$ \\
\hline Assaí (Euterpe oleracea Mart.) & 18.83 & 0 & 0 & 37.17 & $0.07 \mathrm{c}$ & NH \\
\hline Mulberry (Rubus sp.) & 95.08 & 2 & 1 & 56,79 & $0.54 \mathrm{c}$ & $\mathrm{PH}$ \\
\hline Fig (Ficus carica L.) 'Roxo de Valinhos' & 94.08 & 4 & 4 & 761 & $7.16 \mathrm{~b}$ & $\mathrm{GH}$ \\
\hline Jabuticaba (Myrciaria jaboticaba (Vell.) Berg) 'Sabará' & 58.50 & 2.50 & 1 & 47.86 & $0.28 \mathrm{c}$ & $\mathrm{PH}$ \\
\hline Guava 'Paluma' (Psidium guajava L.) susceptible control. & 186 & 5 & 5 & 11,742 & $218.4 \mathrm{a}$ & $\mathrm{GH}$ \\
\hline \multicolumn{7}{|l|}{ Cashew (Anacardium occidentale L.) } \\
\hline 'CCP 06’ & 51.5 & 0 & 0 & 0 & $0 \mathrm{~b}$ & $\mathrm{NH}$ \\
\hline 'CCP 1001’ & 53 & 0 & 0 & 0 & $0 \mathrm{~b}$ & NH \\
\hline \multicolumn{7}{|l|}{ Mango (Mangifera indica L.) } \\
\hline 'Comum’ & 52 & 0 & 0 & 0 & $0 \mathrm{~b}$ & $\mathrm{NH}$ \\
\hline 'Coquinho' & 33.50 & 0 & 0 & 0 & $0 \mathrm{~b}$ & NH \\
\hline 'Espada' & 90.50 & 0 & 0 & 0 & $0 \mathrm{~b}$ & $\mathrm{NH}$ \\
\hline \multicolumn{7}{|l|}{ Papaya (Carica papaya L.) } \\
\hline 'Formosa' & 28.79 & 3.43 & 2.86 & 10.42 & $0.03 \mathrm{~b}$ & $\mathrm{NH}$ \\
\hline 'Papaya' & 46.06 & 4.63 & 3.88 & 17.37 & $0.08 \mathrm{~b}$ & NH \\
\hline Guava 'Paluma' (Psidium guajava L.), susceptible control. & 250 & 5 & 5 & 2360 & $59 \mathrm{a}$ & $\mathrm{GH}$ \\
\hline \multicolumn{7}{|l|}{ Banana (Musa spp.) } \\
\hline (AAB/maçã) ‘Thap Maeo’ & 282 & 4.86 & 4.71 & 1308 & $36.88 \mathrm{~b}$ & $\mathrm{GH}$ \\
\hline (AAB/ plátano) 'Terra' & 137 & 5 & 4.29 & 2328 & $31.90 \mathrm{~b}$ & $\mathrm{GH}$ \\
\hline (AAAB/ maçã) 'Princesa' & 380 & 4.86 & 4.43 & 458 & $17.40 \mathrm{~b}$ & $\mathrm{GH}$ \\
\hline (AAAB/maçã) 'Tropical' & 385 & 4.6 & 4 & 401 & $15.40 \mathrm{~b}$ & $\mathrm{GH}$ \\
\hline (AAAB/prata) 'Garantida' & 314 & 5 & 4.9 & 452 & $14.20 \mathrm{~b}$ & $\mathrm{GH}$ \\
\hline (AAAB/ prata) 'Galil 18' & 371 & 5 & 5 & 373 & $13.83 \mathrm{~b}$ & $\mathrm{GH}$ \\
\hline (AAB/ prata) 'Prata anã' & 342 & 5 & 5 & 335 & $11.47 \mathrm{~b}$ & $\mathrm{GH}$ \\
\hline (AAAB/ prata) 'Japira' & 316 & 4.88 & 4.63 & 280 & $8.84 \mathrm{c}$ & $\mathrm{GH}$ \\
\hline (AAAB/ prata) 'Preciosa' & 330 & 3.63 & 2.88 & 207 & $6.84 \mathrm{c}$ & $\mathrm{GH}$ \\
\hline (AA/cavendish)‘Grande Naine’ & 248 & 4.50 & 3.38 & 256 & $6.35 \mathrm{c}$ & $\mathrm{GH}$ \\
\hline Guava 'Paluma' Psidium guajava L. susceptible control & 320 & 5 & 5 & 2231 & $71.4 \mathrm{a}$ & $\mathrm{GH}$ \\
\hline
\end{tabular}

Data were transformed as $\log (\mathrm{x}+1)$. Means followed by different letters in the column are significantly different according to Scott-Knot's test $(P<0.05)$. Coefficient of variation $(\mathrm{CV})=27 \% .31 \%, 29 \%$

${ }^{1}$ Mean values $(n=8)$ of fresh root weight (FRW)

${ }^{2}$ Mean values $(n=8)$ of gall index and egg mass index, based on a $0-5$ scale, where 0 : no gall/egg mass, 1: 1-2 galls/egg mass, 2: 3-10 galls/ egg mass, 3: 11-30 galls/egg mass, 4: 31-100 galls/egg mass and 5: > 100 galls /egg mass (Hartman \& Sasser 1985)

${ }^{3}$ Mean values $(n=8)$ of number of eggs per gram of roots

${ }^{4}$ Reproduction factor $(\mathrm{RF}=$ final population/10,000 eggs of $M$. enterolobii)

${ }^{5} \mathrm{R}=$ reaction of inoculated plants: $\mathrm{RF}<0.1=$ non-host $(\mathrm{NH}), 0.1<\mathrm{RF}<1=$ poor host $(\mathrm{PH})$ and $\mathrm{RF} \geq 1=\operatorname{good}$ host $(\mathrm{GH})($ Sasser et al. 1984)

carried out in 2012-2013 (Tables 2, 4, 6 and 8). GI and EMI were evident on all good hosts (GI and EMI >2.0) and poor and non hosts (GI and EMI < 2.0), and they were used mostly to describe root damage. The reproduction factors (RF) were used to determine the host reactions of different plants.
One fig genotype, ten banana genotypes (RF $\sim 6.35$ to 36.88) (Tables 3 and 4), six melons ( $R F \sim 2.61$ to 8.58) and six Barbados cherries (Tables 1 and 2) were good-hosts for $M$. enterolobii. Seven strawberry genotypes (Tables 1 and 2), one assaí, two cashews, two papayas, three mangoes (Tables 3 and 4), one avocado, 
Table 4 Host status of fruit crops to Meloidogyne enterolobii under controlled conditions six months after nematode inoculation carried out in 2012 and 2013

\begin{tabular}{|c|c|c|c|c|c|c|}
\hline Fruit crops and genotypes & FRW $(g)^{1}$ & $\mathrm{GI}^{2}$ & $\mathrm{EMI}^{2}$ & $\mathrm{NE} / \mathrm{g}_{\text {roots }}{ }^{3}$ & $\mathrm{RF}^{4}$ & Reaction $^{5}$ \\
\hline Assaí (Euterpe oleracea Mart.) & 28.17 & 0 & 0 & 17.74 & $0.05 \mathrm{c}$ & NH \\
\hline Mulberry (Rubus sp.) & 54.20 & 0 & 0 & 55.35 & $0.30 \mathrm{c}$ & $\mathrm{PH}$ \\
\hline Fig (Ficus carica L.) 'Roxo de Valinhos' & 88.78 & 4 & 4 & 1144 & $10.16 b$ & $\mathrm{GH}$ \\
\hline Jabuticaba (Myrciaria jaboticaba (Vell.) Berg) 'Sabará' & 75.50 & 1 & 1 & 46.36 & $0.35 \mathrm{c}$ & $\mathrm{PH}$ \\
\hline Guava 'Paluma' (Psidium guajava L.) susceptible control. & 124 & 5 & 5 & 10,080 & $125 \mathrm{a}$ & $\mathrm{GH}$ \\
\hline \multicolumn{7}{|l|}{ Cashew (Anacardium occidentale L.) } \\
\hline 'CCP 06’ & 65.3 & 0 & 0 & 0 & $0 \mathrm{~b}$ & NH \\
\hline 'CCP 1001' & 58.4 & 0 & 0 & 0 & $0 \mathrm{~b}$ & $\mathrm{NH}$ \\
\hline \multicolumn{7}{|l|}{ Mango (Mangifera indica L.) } \\
\hline 'Comum’ & 59.8 & 0 & 0 & 0 & $0 \mathrm{~b}$ & $\mathrm{NH}$ \\
\hline 'Coquinho’ & 53.6 & 0 & 0 & 0 & $0 \mathrm{~b}$ & $\mathrm{NH}$ \\
\hline 'Espada' & 60.4 & 0 & 0 & 0 & $0 \mathrm{~b}$ & NH \\
\hline \multicolumn{7}{|l|}{ Papaya (Carica papaya L.) } \\
\hline 'Formosa' & 58.28 & 4.0 & 2.26 & 8,58 & $0.05 \mathrm{~b}$ & $\mathrm{NH}$ \\
\hline 'Papaya' & 54.06 & 4.8 & 2.90 & 18.50 & $0.10 \mathrm{~b}$ & $\mathrm{NH}$ \\
\hline Guava 'Paluma' (Psidium guajava L.), susceptible control & 232 & 5 & 5 & 5474 & $127 \mathrm{a}$ & $\mathrm{GH}$ \\
\hline \multicolumn{7}{|l|}{ Banana (Musa spp.) } \\
\hline (AAB/maçã) ‘Thap Maeo’ & 254 & 5 & 5 & 1094 & $27.80 \mathrm{~b}$ & $\mathrm{GH}$ \\
\hline (AAB/ plátano) 'Terra' & 237 & 5 & 5 & 1384 & $32.80 \mathrm{~b}$ & $\mathrm{GH}$ \\
\hline (AAAB/ maçã) 'Princesa' & 325 & 5 & 5 & 841 & $27.32 \mathrm{~b}$ & $\mathrm{GH}$ \\
\hline (AAAB/maçã) 'Tropical' & 284 & 5 & 5 & 648 & $18.40 \mathrm{~b}$ & $\mathrm{GH}$ \\
\hline (AAAB/prata) 'Garantida' & 325 & 5 & 5 & 775 & $25.20 \mathrm{~b}$ & $\mathrm{GH}$ \\
\hline (AAAB/ prata) 'Galil 18' & 368 & 5 & 5 & 577 & $21.23 \mathrm{~b}$ & $\mathrm{GH}$ \\
\hline (AAB/ prata) 'Prata anã' & 284 & 5 & 5 & 611 & $17.36 \mathrm{~b}$ & $\mathrm{GH}$ \\
\hline (AAAB/ prata) 'Japira' & 328 & 5 & 5 & 867 & $28.44 \mathrm{~b}$ & $\mathrm{GH}$ \\
\hline (AAAB/ prata) 'Preciosa' & 366 & 5 & 5 & 442 & $16.18 \mathrm{~b}$ & $\mathrm{GH}$ \\
\hline (AA/cavendish)'Grande Naine’ & 352 & 5 & 5 & 973 & $34.25 \mathrm{~b}$ & $\mathrm{GH}$ \\
\hline Guava 'Paluma' Psidium guajava L. susceptible control & 280 & 5 & 5 & 7929 & $222 \mathrm{a}$ & GH \\
\hline
\end{tabular}

Data were transformed as $\log (\mathrm{x}+1)$. Means followed by different letters in the column are significantly different according to Scott-Knot's test $(P<0.05)$. Coefficient of variation $(\mathrm{CV})=35 \%, .22 \%, 38 \%$

${ }^{1}$ Mean values $(n=8)$ of fresh root weight (FRW)

${ }^{2}$ Mean values $(n=8)$ of gall index and egg mass index, based on a $0-5$ scale, where 0 : no gall/egg mass, 1: 1-2 galls/egg mass, 2: 3-10 galls/ egg mass, 3: 11-30 galls/egg mass, 4: 31-100 galls/egg mass and 5: > 100 galls /egg mass (Hartman \& Sasser 1985)

${ }^{3}$ Mean values $(n=8)$ of number of eggs per gram of roots

${ }^{4}$ Reproduction factor $(\mathrm{RF}=$ final population/10,000 eggs of $M$. enterolobii)

${ }^{5} \mathrm{R}=$ reaction of inoculated plants: $\mathrm{RF}<0.1=$ non-host $(\mathrm{NH}), 0.1<\mathrm{RF}<1=$ poor host $(\mathrm{PH})$ and $\mathrm{RF} \geq 1=\operatorname{good}$ host $(\mathrm{GH})($ Sasser et al. 1984)

one atemoya, two soursops, three sapodillas and three coconuts (Tables 5 and 6) behaved as nonhosts to $M$. enterolobii. All citrus species and cultivars were considered non-hosts and nine starfruit genotypes were either non-hosts or poor- hosts (Tables 5 and 6). One mulberry and one jabuticaba cultivar behaved as poor hosts to M. enterolobii (Tables 3 and 4). All twelve passion fruit genotypes tested behaved either as non-hosts or poor-hosts (Tables 7 and 8). There was a 
Table 5 Host status of fruit crops to Meloidogyne enterolobii under controlled conditions eight months after nematode inoculation carried out in 2010-2011

\begin{tabular}{|c|c|c|c|c|c|c|}
\hline Fruit crops and genotypes & FRW $(g)^{1}$ & $\mathrm{GI}^{2}$ & $\mathrm{EMI}^{2}$ & $\mathrm{NE} / \mathrm{g}$ roots & $\mathrm{RF}^{4}$ & Reaction $^{5}$ \\
\hline Avocado (Persea americana Mill.) 'Antillean race' & 795 & 0 & 0 & 0 & $0 \mathrm{~b}$ & NH \\
\hline Atemoya (Annona cherimola Mill. x A. squamosa L.) 'Orgulho Africano’ & 75 & 0 & 0 & 2.67 & $0.02 \mathrm{~b}$ & $\mathrm{NH}$ \\
\hline \multicolumn{7}{|l|}{ Soursoup (Annona muricata L.) } \\
\hline 'Comum’ & 22.79 & 0 & 0 & 0.67 & $0 \mathrm{~b}$ & NH \\
\hline 'Morada' & 25.75 & 0 & 0 & 0.24 & $0 \mathrm{~b}$ & $\mathrm{NH}$ \\
\hline \multicolumn{7}{|l|}{ Sapodilla (Manilkara zapota L.) } \\
\hline 'IPA 180’ & 9.50 & 0.13 & 0.13 & 21 & $0.02 \mathrm{~b}$ & $\mathrm{NH}$ \\
\hline 'IPA 31' & 9.00 & 0.13 & 0.13 & 11 & $0.01 \mathrm{~b}$ & NH \\
\hline 'IPA 33' & 8.50 & 0 & 0 & 0 & $0 \mathrm{~b}$ & $\mathrm{NH}$ \\
\hline Guava 'Paluma' (Psidium guajava L.) - susceptible control. & 245 & 5 & 5 & 12,122 & $297 \mathrm{a}$ & $\mathrm{GH}$ \\
\hline \multicolumn{7}{|l|}{ Star fruit (Averrhoa carambola) } \\
\hline 'Hart' & 130 & 0 & 0 & 13.85 & $0.18 \mathrm{~b}$ & $\mathrm{PH}$ \\
\hline 'Arkin' & 143 & 0 & 0 & 7.69 & $0.11 \mathrm{~b}$ & $\mathrm{PH}$ \\
\hline 'Tean-ma' & 188 & 0 & 0 & 4.25 & $0.08 \mathrm{~b}$ & $\mathrm{NH}$ \\
\hline 'Weller' & 133 & 0 & 0 & 3.76 & $0.05 \mathrm{~b}$ & $\mathrm{NH}$ \\
\hline 'Star King Sweet' & 122 & 0 & 0 & 2.46 & $0.03 \mathrm{~b}$ & $\mathrm{NH}$ \\
\hline 'Butts' & 47 & 0 & 0 & 0 & $0 \mathrm{~b}$ & $\mathrm{NH}$ \\
\hline 'Fwang Tung' & 93 & 0 & 0 & 0 & $0 \mathrm{~b}$ & $\mathrm{NH}$ \\
\hline 'Golden Star' & 175 & 0 & 0 & 0 & $0 \mathrm{~b}$ & $\mathrm{NH}$ \\
\hline 'Nota-10' & 108 & 0 & 0 & 0 & $0 \mathrm{~b}$ & $\mathrm{NH}$ \\
\hline \multicolumn{7}{|l|}{ Coconut (Cocos nucifera L.) } \\
\hline 'Anão verde' & 83.5 & 0 & 0 & 7.19 & $0.06 \mathrm{~b}$ & $\mathrm{NH}$ \\
\hline 'BR 001' & 84.75 & 0 & 0 & 0 & $0 \mathrm{~b}$ & $\mathrm{NH}$ \\
\hline 'Gigante do Brasil da Praia Forte' & 40.92 & 0 & 0 & 4.88 & $0.02 \mathrm{~b}$ & $\mathrm{NH}$ \\
\hline Guava 'Paluma' (Psidium guajava L.) - susceptible control. & 250 & 5 & 5 & 2360 & 59 a & $\mathrm{GH}$ \\
\hline \multicolumn{7}{|l|}{ Citrus } \\
\hline Citrange (C. sinensis [L.] Osb. x Poncirus trifoliata [L.] Raf.)'Troyer' & 8 & 0 & 0 & 5.06 & $0 \mathrm{~b}$ & $\mathrm{NH}$ \\
\hline Citrange 'Carrizo' & 10.69 & 0 & 0 & 0 & $0 \mathrm{~b}$ & $\mathrm{NH}$ \\
\hline Lemon (Citrus volkameriana Pasq.) ‘Volkameriano’ & 14.69 & 0 & 0 & 6.80 & $0.01 \mathrm{~b}$ & $\mathrm{NH}$ \\
\hline Citrumelo (C. paradisi Macf. $\times$ P. trifoliata)'Swingle' & 7.69 & 0 & 0 & 0 & $0 \mathrm{~b}$ & $\mathrm{NH}$ \\
\hline Lemon (C. limonia Osbeck) 'Cravo' & 22 & 0 & 0 & 0 & $0 \mathrm{~b}$ & $\mathrm{NH}$ \\
\hline Tangerine (C. reticulata Blanco) 'Cleópatra' & 2.63 & 0 & 0 & 0 & $0 \mathrm{~b}$ & $\mathrm{NH}$ \\
\hline Tangerine (C. sunki Hort. Ex Tan.) 'Sunki’ & 7.44 & 0 & 0 & 0 & $0 \mathrm{~b}$ & $\mathrm{NH}$ \\
\hline Trifoliata (Poncirus trifoliata) & 3.06 & 0 & 0 & 0 & $0 \mathrm{~b}$ & $\mathrm{NH}$ \\
\hline Guava 'Paluma' (Psidium guajava L.) - susceptible control & 301 & 5 & 5 & 12,193 & 367 a & $\mathrm{GH}$ \\
\hline
\end{tabular}

Data were transformed as $\log (\mathrm{x}+1)$. Means followed by different letters in the column are significantly different according to Scott-Knot's test $(P<0.05)$. Coefficient of variation $(\mathrm{CV})=35 \%, 24 \%, 32 \%$

${ }^{1}$ Mean values $(n=8)$ of fresh root weight (FRW)

${ }^{2}$ Mean values $(n=8)$ of gall index and egg mass index, based on a $0-5$ scale, where $0:$ no gall/egg mass, 1: 1-2 galls/egg mass, $2: 3-10$ galls/ egg mass, 3: 11-30 galls/egg mass, 4: 31-100 galls/egg mass and 5: > 100 galls /egg mass (Hartman \& Sasser 1985)

${ }^{3}$ Mean values $(n=8)$ of number of eggs per gram of roots

${ }^{4}$ Reproduction factor $(\mathrm{RF}=$ final population/10,000 eggs of $M$. enterolobii)

${ }^{5} \mathrm{R}=$ reaction of inoculated plants: $\mathrm{RF}<0.1=$ non-host $(\mathrm{NH}), 0.1<\mathrm{RF}<1=$ poor host $(\mathrm{PH})$ and $\mathrm{RF} \geq 1=\operatorname{good}$ host $(\mathrm{GH})($ Sasser et al. 1984) 
Table 6 Host status of fruit crops to Meloidogyne enterolobii under controlled conditions eight months after nematode inoculation carried out in 2012-2013

\begin{tabular}{|c|c|c|c|c|c|c|}
\hline Fruit crops and genotypes & FRW $(g)^{1}$ & $\mathrm{GI}^{2}$ & $\mathrm{EMI}^{2}$ & $\mathrm{NE} / \mathrm{g}$ roots & $\mathrm{RF}^{4}$ & Reaction $^{5}$ \\
\hline Avocado (Persea americana Mill.) 'Antillean race' & 642 & 0 & 0 & 0 & $0 \mathrm{~b}$ & NH \\
\hline Atemoya (Annona cherimola Mill. x A. squamosa L.) 'Orgulho Africano’ & 94 & 0 & 0 & 0 & $0 \mathrm{~b}$ & $\mathrm{NH}$ \\
\hline \multicolumn{7}{|l|}{ Soursoup (Annona muricata L.) } \\
\hline 'Comum’ & 31.64 & 0 & 0 & 0 & $0 \mathrm{~b}$ & NH \\
\hline 'Morada' & 28.26 & 0 & 0 & 0 & $0 \mathrm{~b}$ & $\mathrm{NH}$ \\
\hline \multicolumn{7}{|l|}{ Sapodilla (Manilkara zapota L.) } \\
\hline 'IPA 180’ & 12.50 & 0 & 0 & 0 & $0 \mathrm{~b}$ & $\mathrm{NH}$ \\
\hline 'IPA 31' & 10.20 & 0 & 0 & 0 & $0 \mathrm{~b}$ & NH \\
\hline 'IPA 33' & 7,86 & 0 & 0 & 0 & $0 \mathrm{~b}$ & $\mathrm{NH}$ \\
\hline Guava 'Paluma' (Psidium guajava L.) - susceptible control & 189 & 5 & 5 & 8042 & $152 \mathrm{a}$ & $\mathrm{GH}$ \\
\hline \multicolumn{7}{|l|}{ Star fruit (Averrhoa carambola) } \\
\hline 'Hart' & 122 & 0 & 0 & 0 & $0 \mathrm{~b}$ & $\mathrm{PH}$ \\
\hline 'Arkin' & 98 & 0 & 0 & 0 & $0 \mathrm{~b}$ & $\mathrm{PH}$ \\
\hline 'Tean-ma' & 164 & 0 & 0 & 0 & $0 \mathrm{~b}$ & $\mathrm{NH}$ \\
\hline 'Weller' & 145 & 0 & 0 & 0 & $0 \mathrm{~b}$ & $\mathrm{NH}$ \\
\hline 'Star King Sweet' & 132 & 0 & 0 & 0 & $0 \mathrm{~b}$ & $\mathrm{NH}$ \\
\hline 'Butts' & 146 & 0 & 0 & 0 & $0 \mathrm{~b}$ & $\mathrm{NH}$ \\
\hline 'Fwang Tung' & 124 & 0 & 0 & 0 & $0 \mathrm{~b}$ & $\mathrm{NH}$ \\
\hline 'Golden Star' & 188 & 0 & 0 & 0 & $0 \mathrm{~b}$ & NH \\
\hline 'Nota-10' & 124 & 0 & 0 & 0 & $0 \mathrm{~b}$ & $\mathrm{NH}$ \\
\hline \multicolumn{7}{|l|}{ Coconut (Cocos nucifera L.) } \\
\hline 'Anão verde' & 53.8 & 0 & 0 & 7.43 & $0.04 \mathrm{~b}$ & $\mathrm{NH}$ \\
\hline 'BR 001' & 88.72 & 0 & 0 & 6.76 & $0.06 \mathrm{~b}$ & $\mathrm{NH}$ \\
\hline 'Gigante do Brasil da Praia Forte' & 70.24 & 0 & 0 & 2.69 & $0.02 \mathrm{~b}$ & $\mathrm{NH}$ \\
\hline Guava 'Paluma' (Psidium guajava L.) - susceptible control & 145 & 5 & 5 & 10,552 & $153 \mathrm{a}$ & GH \\
\hline \multicolumn{7}{|l|}{ Citrus } \\
\hline Citrange (C. sinensis [L.] Osb. x Poncirus trifoliata [L.] Raf.)'Troyer' & 12.24 & 0 & 0 & 0 & $0 \mathrm{~b}$ & $\mathrm{NH}$ \\
\hline Citrange 'Carrizo' & 15.24 & 0 & 0 & 0 & $0 \mathrm{~b}$ & $\mathrm{NH}$ \\
\hline Lemon (Citrus volkameriana Pasq.) 'Volkameriano’ & 16.44 & 0 & 0 & 0 & $0 \mathrm{~b}$ & $\mathrm{NH}$ \\
\hline Citrumelo (C. paradisi Macf. $\times$ P. trifoliata)'Swingle’ & 10.26 & 0 & 0 & 0 & $0 \mathrm{~b}$ & $\mathrm{NH}$ \\
\hline Lemon (C. limonia Osbeck) ‘Cravo’ & 28.50 & 0 & 0 & 0 & $0 \mathrm{~b}$ & $\mathrm{NH}$ \\
\hline Tangerine (C. reticulata Blanco) 'Cleópatra' & 22.33 & 0 & 0 & 0 & $0 \mathrm{~b}$ & $\mathrm{NH}$ \\
\hline Tangerine (C. sunki Hort. Ex Tan.) 'Sunki’ & 17.24 & 0 & 0 & 0 & $0 \mathrm{~b}$ & $\mathrm{NH}$ \\
\hline Trifoliata (Poncirus trifoliata) & 23.08 & 0 & 0 & 0 & $0 \mathrm{~b}$ & $\mathrm{NH}$ \\
\hline Guava 'Paluma' (Psidium guajava L.) - susceptible control & 225 & 5 & 5 & 11,111 & $250 \mathrm{a}$ & $\mathrm{GH}$ \\
\hline
\end{tabular}

Data were transformed as $\log (\mathrm{x}+1)$. Means followed by different letters in the column are significantly different according to Scott-Knot's test $(P<0.05)$. Coefficient of variation $(\mathrm{CV})=22 \%, 24 \%, 20 \%$

${ }^{1}$ Mean values $(n=8)$ of fresh root weight (FRW)

${ }^{2}$ Mean values $(n=8)$ of gall index and egg mass index, based on a $0-5$ scale, where 0 : no gall/egg mass, 1: 1-2 galls/egg mass, 2: 3-10 galls/ egg mass, 3: 11-30 galls/egg mass, 4: 31-100 galls/egg mass and 5: > 100 galls /egg mass (Hartman \& Sasser 1985)

${ }^{3}$ Mean values $(n=8)$ of number of eggs per gram of roots

${ }^{4}$ Reproduction factor $(\mathrm{RF}=$ final population/10,000 eggs of $M$. enterolobii)

${ }^{5} \mathrm{R}=$ reaction of inoculated plants: $\mathrm{RF}<0.1=$ non-host $(\mathrm{NH}), 0.1<\mathrm{RF}<1=$ poor host $(\mathrm{PH})$ and $\mathrm{RF} \geq 1=\operatorname{good}$ host $(\mathrm{GH})(\mathrm{Sasser}$ et al. 1984) 
Table 7 Host status of fruit crops to Meloidogyne enterolobii under controlled conditions height months after nematode inoculation carried out in 2010-2011

\begin{tabular}{|c|c|c|c|c|c|c|}
\hline Fruit crops and genotypes & $\operatorname{FRW}(\mathrm{g})^{1}$ & $\mathrm{GI}^{2}$ & $\mathrm{EMI}^{2}$ & $\mathrm{NE} / \mathrm{g} \mathrm{roots}^{3}$ & $\mathrm{RF}^{4}$ & Reaction $^{5}$ \\
\hline \multicolumn{7}{|l|}{ Passion fruit (Passiflora spp.) } \\
\hline Sweet passion fruit (P. nitida Kunth.) & 31.50 & 0.75 & 0.75 & 156 & $0.49 \mathrm{~b}$ & $\mathrm{PH}$ \\
\hline Wild passion fruit (P. setacea D.C.) & 36.60 & 0 & 0 & 76.50 & $0.28 \mathrm{~b}$ & $\mathrm{PH}$ \\
\hline Sweet passion fruit 'Decaisneana' (P. alata $\mathrm{x} P$. quadrangularis) & 36.25 & 1 & 0 & 35.86 & $0.13 \mathrm{~b}$ & $\mathrm{PH}$ \\
\hline Wild passion fruit with red flower (P. coccinea Aubl.) & 77.88 & 0 & 0 & 15.41 & $0.12 \mathrm{~b}$ & $\mathrm{PH}$ \\
\hline Ornamental passion fruit 'Estrela do cerrado' ( $P$. coccinia $\mathrm{x}$ P. setacea $)$ & 25.63 & 0 & 0 & 39.02 & $0.10 \mathrm{~b}$ & $\mathrm{PH}$ \\
\hline Hybrid $P$. edulis x $P$. Setacea & 60.83 & 0 & 0 & 16.44 & $0.10 \mathrm{~b}$ & $\mathrm{PH}$ \\
\hline Wild passion fruit ( $P$. cincinata Mast.) & 35.44 & 0.13 & 0 & 22.57 & $0.08 \mathrm{~b}$ & $\mathrm{NH}$ \\
\hline Sour passion fruit 'Vermelhinho pequeno' (hybrid P. edulis f. flavicarpa) & 33.63 & 0 & 0 & 26.76 & $0.09 \mathrm{~b}$ & $\mathrm{NH}$ \\
\hline Ornamental passion fruit 'Rubiflora' ( $P$. coccinea $\times$ P. setacea $)$ & 43.12 & 0.25 & 0 & 0 & $0 \mathrm{~b}$ & $\mathrm{NH}$ \\
\hline Sour passion fruit 'Gigante Amarelo' - GA2 (hybrid P. edulis f. flavicarpa) & 105 & 0.25 & 0 & 0 & $0 \mathrm{~b}$ & $\mathrm{NH}$ \\
\hline Sour passion fruit 'Sol do Cerrado' - EC2 (hybrid $P$. edulis f. flavicarpa) & 84.38 & 0 & 0 & 0 & $0 \mathrm{~b}$ & $\mathrm{NH}$ \\
\hline Sour passion fruit 'Ouro vermelho' - AR1 (hybrid P. edulis f. flavicarpa) & 33.08 & 0 & 0 & 0 & $0 \mathrm{~b}$ & NH \\
\hline Guava 'Paluma' (Psidium guajava L.) - susceptible control. & 390 & 5 & 5 & 7231 & $282 \mathrm{a}$ & GH \\
\hline \multicolumn{7}{|l|}{ Grape (Vitis spp.) } \\
\hline ‘Chardonnay’ & 13.35 & 4.33 & 4.33 & 3633 & $4.85 \mathrm{~b}$ & $\mathrm{GH}$ \\
\hline 'Solferino' & 18.25 & 4.33 & 4.33 & 619 & $1.13 \mathrm{c}$ & $\mathrm{GH}$ \\
\hline 'IAC 766' & 11.57 & 1 & 1 & 86.43 & $0.10 \mathrm{c}$ & $\mathrm{PH}$ \\
\hline 'IAC 572' & 22.47 & 1.33 & 1.33 & 26.70 & $0.06 \mathrm{c}$ & $\mathrm{NH}$ \\
\hline 'Rupestris du Lot' & 16.92 & 1 & 1 & 29.55 & $0.05 \mathrm{c}$ & $\mathrm{NH}$ \\
\hline 'IAC313' & 20.06 & 0.33 & 0.33 & 14.95 & $0.03 \mathrm{c}$ & $\mathrm{NH}$ \\
\hline 'Paulsen 1103' & 25.57 & 0.80 & 0.80 & 11.73 & $0.03 \mathrm{c}$ & $\mathrm{NH}$ \\
\hline 'Salt Creek' & 8.60 & 0 & 0 & 11.62 & $0.01 \mathrm{c}$ & $\mathrm{NH}$ \\
\hline 'Harmony' & 30.02 & 0 & 0 & 0 & $0 \mathrm{c}$ & $\mathrm{NH}$ \\
\hline 'K5BB -Kobber' & 11.64 & 0 & 0 & 0 & $0 \mathrm{c}$ & $\mathrm{NH}$ \\
\hline Tomato 'Santa Clara' (Solanum lycopersicum L.) - susceptible control. & 39.50 & 5 & 5 & 9939 & $39.26 \mathrm{a}$ & GH \\
\hline
\end{tabular}

Data were transformed as $\log (\mathrm{x}+1)$. Means followed by different letters in the column are significantly different according to Scott-Knot's test $(P<0.05)$. Coefficient of variation $(\mathrm{CV})=27 \%, 29 \%$

${ }^{1}$ Mean values $(n=8)$ of fresh root weight (FRW)

${ }^{2}$ Mean values $(n=8)$ of gall index and egg mass index, based on a $0-5$ scale, where $0:$ no gall/egg mass, 1: 1-2 galls/egg mass, $2: 3-10$ galls/ egg mass, 3: 11-30 galls/egg mass, 4: 31-100 galls/egg mass and 5: > 100 galls /egg mass (Hartman \& Sasser 1985)

${ }^{3}$ Mean values $(n=8)$ of number of eggs per gram of roots

${ }^{4}$ Reproduction factor $(\mathrm{RF}=$ final population/10,000 eggs of $M$. enterolobii)

${ }^{5} \mathrm{R}=$ reaction of inoculated plants: $\mathrm{RF}<0.1=$ non-host $(\mathrm{NH}), 0.1<\mathrm{RF}<1=$ poor host $(\mathrm{PH})$ and $\mathrm{RF} \geq 1=\operatorname{good}$ host $(\mathrm{GH})(\mathrm{Sasser}$ et al. 1984)

variation in host suitability of grape genotype rootstocks against $M$. enterolobii: two genotypes behaved as non-hosts, six as poor hosts and two as good hosts: 'Solferino' and 'Chardonnay' (Tables 7 and 8). All susceptible guava and tomato controls behaved as good hosts to $M$. enterolobii (Tables 1, 2, 3, 4, 5, 6, 7 and 8).

\section{Discussion}

Commercial guava orchards have been destroyed in some countries in Central and South America by M. enterolobii. To date, no effective control methods have been identified for this damaging nematode species in guava (Carneiro et al. 2012). Psidium guajava cv. 
Table 8 Host status of fruit crops to Meloidogyne enterolobii under controlled conditions eight months after nematode inoculation carried out in 2012-2013

\begin{tabular}{|c|c|c|c|c|c|c|}
\hline Fruit crops and genotypes & FRW $(g)^{1}$ & $\mathrm{GI}^{2}$ & $\mathrm{EMI}^{2}$ & $\mathrm{NE} / \mathrm{g} \mathrm{roots}^{3}$ & $\mathrm{RF}^{4}$ & Reaction $^{5}$ \\
\hline \multicolumn{7}{|l|}{ Passion fruit (Passiflora spp.) } \\
\hline Sweet passion fruit (P. nitida Kunth.) & 63.40 & 0 & 0 & 0 & $0 \mathrm{~b}$ & $\mathrm{NH}$ \\
\hline Wild passion fruit (P. setacea D.C.) & 48.60 & 0 & 0 & 0 & $0 \mathrm{~b}$ & $\mathrm{NH}$ \\
\hline Sweet passion fruit 'Decaisneana' ( $P$. alata $\times$ P. quadrangularis $)$ & 45.20 & 0 & 0 & 0 & $0 \mathrm{~b}$ & NH \\
\hline Wild passion fruit with red flower (P. coccinea Aubl.) & 67.50 & 0 & 0 & 0 & $0 \mathrm{~b}$ & $\mathrm{NH}$ \\
\hline Ornamental passion fruit 'Estrela do cerrado' $(P$. coccinia $\mathrm{x} P$. setacea $)$ & 35.33 & 0 & 0 & 0 & $0 \mathrm{~b}$ & NH \\
\hline Hybrid P. edulis x P. Setacea & 58.24 & 0 & 0 & 0 & $0 \mathrm{~b}$ & NH \\
\hline Wild passion fruit ( $P$. cincinata Mast.) & 38.32 & 0 & 0 & 0 & $0 \mathrm{~b}$ & NH \\
\hline Sour passion fruit 'Vermelhinho pequeno' (hybrid P. edulis f. flavicarpa) & 46.28 & 0 & 0 & 0 & $0 \mathrm{~b}$ & $\mathrm{NH}$ \\
\hline Ornamental passion fruit 'Rubiflora' ( $P$. coccinea $\times$ P. setacea $)$ & 39.27 & 0 & 0 & 0 & $0 \mathrm{~b}$ & $\mathrm{NH}$ \\
\hline Sour passion fruit 'Gigante Amarelo' - GA2 (hybrid P. edulis f. flavicarpa) & 85.40 & 0 & 0 & 0 & $0 \mathrm{~b}$ & $\mathrm{NH}$ \\
\hline Sour passion fruit 'Sol do Cerrado' - EC2 (hybrid $P$. edulis f. flavicarpa) & 83.24 & 0 & 0 & 0 & $0 \mathrm{~b}$ & $\mathrm{NH}$ \\
\hline Sour passion fruit 'Ouro vermelho' - AR1 (hybrid P. edulis f. flavicarpa) & 48.34 & 0 & 0 & 0 & $0 \mathrm{~b}$ & NH \\
\hline Guava 'Paluma' (Psidium guajava L.) - susceptible control. & 192 & 5 & 5 & 8594 & $165 \mathrm{a}$ & GH \\
\hline \multicolumn{7}{|l|}{ Grape (Vitis spp.) } \\
\hline 'Chardonnay' & 23.25 & 4.33 & 4.33 & 3677 & $8.55 \mathrm{~b}$ & GH \\
\hline 'Solferino' & 22.30 & 4.33 & 4.33 & 1453 & $3.24 \mathrm{~b}$ & $\mathrm{GH}$ \\
\hline 'IAC 766' & 21.75 & 0 & 0 & 18.39 & $0.04 \mathrm{c}$ & $\mathrm{NH}$ \\
\hline 'IAC 572' & 18.25 & 0 & 0 & 43.84 & $0.08 \mathrm{c}$ & $\mathrm{NH}$ \\
\hline 'Rupestris du Lot' & 20.34 & 0 & 0 & 19.67 & $0.04 \mathrm{c}$ & $\mathrm{NH}$ \\
\hline 'IAC313' & 24.23 & 0 & 0 & 24.76 & $0.06 \mathrm{c}$ & $\mathrm{NH}$ \\
\hline 'Paulsen 1103' & 18.57 & 0 & 0 & 21.54 & $0.04 \mathrm{c}$ & $\mathrm{NH}$ \\
\hline 'Salt Creek' & 28,26 & 0 & 0 & 3.54 & $0.01 \mathrm{c}$ & $\mathrm{NH}$ \\
\hline 'Harmony' & 32.08 & 0 & 0 & 6.23 & $0.02 \mathrm{c}$ & $\mathrm{NH}$ \\
\hline 'K5BB -Kobber' & 31.44 & 0 & 0 & 0 & $0 \mathrm{c}$ & $\mathrm{NH}$ \\
\hline Guava 'Paluma' (Psidium guajava L.) - susceptible control & 195 & 5 & 5 & 6256 & $122 \mathrm{a}$ & $\mathrm{GH}$ \\
\hline
\end{tabular}

Data were transformed as $\log (\mathrm{x}+1)$. Means followed by different letters in the column are significantly different according to Scott-Knot's test $(P<0.05)$. Coefficient of variation $(\mathrm{CV})=20 \%, 28 \%$

${ }^{1}$ Mean values $(n=8)$ of fresh root weight (FRW)

${ }^{2}$ Mean values $(n=8)$ of gall index and egg mass index, based on a $0-5$ scale, where $0:$ no gall/egg mass, 1: 1-2 galls/egg mass, $2: 3-10$ galls/ egg mass, 3: 11-30 galls/egg mass, 4: 31-100 galls/egg mass and 5: > 100 galls /egg mass (Hartman \& Sasser 1985)

${ }^{3}$ Mean values $(n=8)$ of number of eggs per gram of roots

${ }^{4}$ Reproduction factor $(\mathrm{RF}=$ final population/10,000 eggs of $M$. enterolobii)

${ }^{5} \mathrm{R}=$ reaction of inoculated plants: $\mathrm{RF}<0.1=$ non-host $(\mathrm{NH}), 0.1<\mathrm{RF}<1=$ poor host $(\mathrm{PH})$ and $\mathrm{RF} \geq 1=\operatorname{good}$ host $(\mathrm{GH})($ Sasser et al. 1984)

Paluma was used as control and showed great variability in reproduction factors $(\mathrm{RF}=59-362)$ in different treatments due to sizes of inoculated plants: smaller plants had lower RFs at the end of the assays due to poor root system caused by $M$. enterolobii infection.

Freitas et al. (2014) assessed 51 Psidium spp. accessions, of which $P$. cattleianum (yellow guava), $P$. friedrichsthalianium (Costa Rican guava), Acca sellowiana (feijoa) and P. rufum (purple guava) were all resistant to $M$. enterolobii, showing a reproduction factor $<1$. All 43 wild accessions of $P$. guajava were susceptible, as well as three accessions of $P$. guineense (Brazilian guava) and one of $P$. acutangulum (pear guava). When used as rootstocks under greenhouse conditions $P$. cattleianum and $P$. friedrichsthalianium were compatible with $P$. guajava cv. Paluma, however, 
in field conditions only the last survived. Considering these results, all commercial and wild P. guajava accessions were considered susceptible to this nematode. Thus, the use of resistant wild Psidium spp. as rootstocks can be considered promising for developing sustainable control methods for M. enterolobii (Freitas et al. 2014). Recently, a hybrid cross between P. guajava $\mathrm{x}$ $P$. guineense has shown resistance to M. enterolobii, and has last for at least five years after transplanting in areas infested with the nematode. In Petrolina state, a major guava producing region in Brazil, this hybrid has been successfully used as rootstock with great compatibility with the guava varieties Paluma and Pedro Sato, showing promising yield of ca. 40 tons of fruits/ ha, compared to 4.5 tons / ha of Paluma ungrafted (Santos et al. 2016).

Another effective control method for this nematode is the use of non-host cover crops. Carneiro et al. (2012) selected 26 different plant species (e.g. peanut, white and black oat, millet, rattlepod, cowpea, castor bean, velvet bean, among others) to be used in a crop rotation scheme in areas infested with $M$. enterolobii, showing an alternative control method for this nematode species.

The results of intraspecific variability among $M$. enterolobii isolates showed a low level of diversity, indicating that this nematode is a genetically homogeneous species (Tigano et al. 2010). Considering this homogeneity, we hypothesize that the use of resistant wild Psidium spp. and crop rotation with different plant species against populations of $M$. enterolobii can be extended to several populations within this species.

In this study, 16 fruit crops behaved as non-hosts or poor hosts to M. enterolobii, including assaí, atemoya, avocado, cashew nut, citrus, coconut, grape, jabuticaba, mango, mulberry, papaya, passion fruit, sapodilla, soursop, starfruit and strawberry; only four fruit plants were good hosts (fig, banana, 'Chardonnay' and 'Solferino' grapes and melon). These results agree with those obtained by Silva and Krasuski (2012) who tested 22 tropical fruit crops of which 16 were immune to M. enterolobii, and disagree from other previously published studies which showed that $M$. enterolobii has a wide host range, parasitizing plants within ten different botanical families: Brassicaceae, Fabaceae, Curcubitaceae, Lamiaceae, Leguminosae, Malvaceae, Chenopodiaceae, Myrtaceae, Solanaceae, and Umbelliferae (Brito et al. 2007).

Few studies have been carried out regarding the parasitism of $M$. enterolobii on tropical fruit crops. Considering that Meloidogyne species frequently occur in mixtures under field conditions (Cofcewicz et al.
2004) and that current research are focused on obtaining plant genotypes and crop rotation with multiple resistance to RKN, we emphasized our discussion considering the management of $M$. enterolobii and other important RKNs. All melon genotypes behaved as good hosts to $M$. enterolobii and are in agreement with those reported by Bitencourt and Silva (2010), who confirmed the susceptibility of yellow melon to this nematode species. Similarly, all banana genotypes behaved as good hosts to M. enterolobii. Usually, all banana cultivars were considered susceptible to main Meloidogyne species (Quénéhervé et al. 2009).

In our study, fig was a good host to M. enterolobii. RKN has been reported to cause damage in fig crop. To date, there are no fig rootstocks resistant to RKN in Brazil (Medina et al. 2006). All Barbados cherry genotypes tested were good hosts to M. enterolobii. Our results were similar to other studies reporting the host suitability of this crop to this nematode species (Souza et al. 2006).

Interestingly, we observed that all passion fruit genotypes behaved as poor hosts to M. enterolobii. However, Lima et al. (2003) reported this nematode species occurring naturally in Passion mucronata Lam., in São João da Barra, Rio de Janeiro state.

The results for host suitability of grape genotype rootstocks against $M$. enterolobii were variable. Two genotypes were rated as non-hosts, six as poor hosts and two as good hosts (cv. Chardonnay and Solferino). The cultivar 'Chardonnay' is known as a standard susceptible rootstock to other RKN (Ingels 1992) and now to $M$. enterolobii as well. Similar to our results, cultivars 'Harmony' (Vitis champinii Planchonx1613c) and 'Paulsen' (V. berlandieri Planch. x V. rupestris Scheele) behaved as resistant to Meloidogyne spp. (Ingels 1992; McKenry and Anwar 2007).

Avocado, atemoya, cashew, soursop and mango were all non-hosts or poor hosts to M. enterolobii. Atemoya, cashew, soursop and sapodilla were considered immune to this nematode by Silva and Krasuski (2012). Multiple resistance to RKN in annonaceous has been reported by other authors (Ponte et al. 1976; Oliveira et al. 1991, Ribeiro et al. 2004).

In this study, all eight citrus species and genotypes tested were non-hosts to M. enterolobii. Similarly, Silva and Krasuski (2012) reported immunity in three Citrus species $(C$. sinensis L Osbeck, $C$. aurantifolia Linn and C. reticulata Blanco). 
We found that all strawberry genotypes were nonhost to $M$. enterolobii. In a similar study, cultivars 'Camarosi' and 'Diamante', studied in this study were also classified as resistant to M. hapla, an important pest in strawberry production worldwide (Pinkerton and Finn 2005).

Mulberry and jabuticaba behaved as poor hosts to M. enterolobii, even though galls were present in infected plants. Contrarily, there are studies reporting the susceptibility of mulberry to other RKN species (Castillo et al. 2001).

Assaí, starfruit, sapodilla and coconut behaved as poor hosts to M. enterolobii. Similarly, Silva and Krasuski (2012) reported immunity in two different sapodillas. There are reports of palm trees and coconuts being infected by other Meloidogyne species in Africa and India (McSorley 1992; Sheela 1995). In addition, other authors reported starfruit and sapodilla as resistant to RKNs (Ponte et al. 1976; Petit 1990).

In this study papaya was non host to M. enterolobii, even though there was gall formation and necrotic egg masses. Our results disagree with those reported by Brito et al. (2008) and Silva and Krasuski (2012), who classified papaya as a host to $M$. enterolobii. Similarly, Siqueira et al. (2009) reported galls of M. enterolobii in papaya fields planted in a consortium: guava x papaya; however, only few egg masses were found in papaya roots collected in the field. These authors concluded in their study that papaya was tolerant to M. enerolobii, since no apparent symptoms were observed and a high fruit yield was obtained.

In conclusion, $M$. enterolobii has been considered a polyphagous species, but we showed that several fruit crops behaved either as non-hosts or poor hosts to this nematode species (e.g. assaí, atemoya, avocado, cashew, citrus, coconut, jabuticaba, mulberry, mango, papaya, passion fruit, sapodilla, soursop, starfruit, strawberry and some grape rootstocks). Our results demonstrated that these fruit species are an option to be planted in areas already infested by $M$. enterolobii after trials in field conditions have been set. In the near future these plant species may replace eradicated guava orchards severely infested by this nematode and become an economic option for growers where $M$. enterolobii is considered a problem.

Acknowledgments Funding for this research was provided by CNPq (Conselho Nacional de Desenvolvimento Científico e Tecnonológico), MAPA (Ministério da Agricultura, Pecuária e
Abastecimento), Embrapa Recursos Genéticos e Biotecnologia and our colleagues who provided the fruit crop genotypes tested in this study.

\section{References}

Bitencourt, N. V., \& Silva, G. S. (2010). Reproduction of Meloidogyne enterolobii on vegetables. Nematologia Brasileira, 34, 181-183.

Brito, J. A., Stanley, J. D., Mendes, M. L., Cetintas, R., \& Dickson, D. W. (2007). Host status of selected cultivated plants to Meloidogyne mayaguensis from Florida. Nematropica, 37, 65-71.

Brito, J. A., Kaur, R., Cetintas, R., Stanley, J. D., Mendes, M. L., Mcavoy, E. J., Powers, T. O., \& Dickson, D. W. (2008). Identification and isozyme characterization of Meloidogyne spp. infecting horticultural and agronomic crops, and weed plants in Florida. Nematology, 10, 757-766.

Carneiro, R. M. D. G., \& Almeida, M. R. A. (2001). Técnica de eletroforese usada no estudo de enzimas dos nematoides de galhas para identificação de espécies. Nematologia Brasileira, 25, 35-44.

Carneiro, R. M. D. G., Moreira, W., Almeida, M. R. A., \& Gomes, A. C. M. M. (2001). Primeiro registro de Meloidogyne mayaguensis em goiabeira no Brasil. Nematologia Brasileira, 25, 223-228.

Carneiro, R. M. D. G., Cirotto, P. A., Silva, D. B., \& Carneiro, R. G. (2007). Resistance to Meloidogyne mayaguensis in Psidium spp. accessions and their grafting compatibility with P. guajava cv. "Paluma". Fitopatologia Brasileira, 32, 281284.

Carneiro, R. M. D. G., Freitas, V. M., Mattos, J. K., Castro, J. M., Gomes, C. B., \& Carneiro, R. G. (2012). Major guava nematodes and control prospects using resistance on Psidium spp. and non-host crops. Acta Horticulturae, 959, 41-49.

Castillo, P., Vito, M., Di Vovlas, N., \& Jimenez-Diaz, R. M. (2001). Host-parasite relationships in root-knot disease of white mulberry. Plant Disease, 85, 277-281.

Cofcewicz, E. T., Carneiro, R. M. D. G., Catagnone-Sereno, P., \& Quénéhervé, P. (2004). Enzyme phenotype and genetic diversity of root-knot nematode parasitizing Musa in Brazil. Nematology, 6, 85-95.

da Silva, G. S., \& Krasuski, A. I. (2012). Reação de algumas espécies frutíferas tropicais a Meloidogyne enterolobii. Nematologia Brasileira, 36, 83-86.

El Borai, F. E., \& Duncan, L. W. (2005). Nematodes parasites of subtropical and tropical fruit tree crops. In M. Luc, R. A. Sikora, \& J. Bridge (Eds.), Plant parasitic nematodes in subtropical and tropical agriculture (pp. 467-492). Wallingford: CAB INTERNATIONAL.

EPPO (2008). An emerging root-knot nematode, Meloidogyne enterolobii: addition to the EPPO Alert List. EPPO Reporting Service, 2008/105.

Freitas, V. M., Correia, V. R., Motta, F. C., Sousa, M. G., Gomes, A. C. M. M., Carneiro, M. D. G., Silva, D. B., Mattos, J. K., Nicole, M., \& Carneiro, R. M. D. G. (2014). Resistant accessions of wild Psidium spp. to Meloidogyne enterolobii 
and histological characterization of resistance. Plant Pathology, 63, 738-746.

Hartman, K. M., \& Sasser, J. N. (1985). Identification of Meloidogyne species on the basis of differential host test and perineal pattern morphology. In K. R. Barker, C. C. Carter, \& J. N. Sasser (Eds.), An advanced treatise on Meloidogyne. Vol. II: methodology (pp. 69-77). Raleigh: North Carolina State University Graphics.

Hussey, R. S., \& Barker, K. R. (1973). A comparison of methods of collecting inocula of Meloidogyne spp., including a new technique. Plant Disease Report, 57, 1025-1028.

Ingels, C. (1992). Sustainable agriculture and grape production. American Journal of Enology and Viticulture, 43, 296-298.

Kiewnick, S., Karssen, G., Brito, J. A., Oggenfuss, M., \& Frey, J. E. (2008). First report of root-knot nematode Meloidogyne enterolobii on tomato and cucumber in Switzerland. Plant Disease, 92, 1370.

Lima, I. M., Dolinki, C. M., \& Souza, R. M. (2003). Dispersão de Meloidogyne mayaguensis em goiabeiras de São João da Barra (RJ) e relato de novos hospedeiros dentre as plantas invasoras e cultivadas. Nematologia Brasileira, 27, 257-258.

Mckenry, M. V., \& Anwar, S. A. (2007). Virulence of Meloidogyne spp. and induced resistance in grape rootstocks. Journal of Nematology, 39, 50-54.

McSorley, R. (1992). Nematological problems in tropical and subtropical fruit tree crops. Nematropica, 22, 103-116.

Medina, I. L., Gomes, C. B., Rossi, C. E., \& Carneiro, R. M. D. G. (2006). Caracterização e identificação de populações de nematoides de galhas provenientes de figueiras (Ficus carica L.) do Rio Grande do Sul e de São Paulo. Nematologia Brasileira, 30, 179-187.

Oliveira, C. M. G., Goulart, A. M. C., Ferraz, L. C. C. B., \& Monteiro, A. R. (1991). Resistência de gravioleira (Annona muricata L.) a Meloidogyne arenaria e M. incognita. Nematologia Brasileira, 15, 204-205.

Pereira, F. O. M., Souza, R. M., Souza, P. M., Dolinski, C., \& Santos, G. K. (2009). Estimativa do impacto econômico e social direto de Meloidogyne mayaguensis na cultura da goiaba no Brasil. Nematologia Brasileira, 33, 176-181.

Petit, R. P. (1990). A survey of plant parasitic nematodes associated with fruit trees of economic importance in Venezuela. Fitopatologia Venezuelana, 3, 2-5.
Pinkerton, J., \& Finn, C. E. (2005). Responses of strawberry species and cultivars to the root-lesion and northern rootknot nematodes. Horticultural Science, 40, 33-38.

Ponte, J. J., Lemos, J. W. V., Castro, F. E., \& Maria, L. (1976). Comportamento de plantas frutíferas tropicais em relação a nematóide das galhas. Fitopatologia Brasileira, 1, 29-33.

Quénéhervé, P., Valette, C., Topart, P., Tezenas Du Montcel, H., \& Salmon, F. (2009). Nematode resistance in bananas: screening results on some wild and cultivated accessions of Musa spp. Euphytica, 165, 123-136.

Ribeiro, R. C. F., Souza, T. H. R., Xavier, A. A., Mizobutsi, E. H., Pereira, F. R., \& Barros, R. F. X. (2004). Reação de diferentes anonáceas a Meloidogyne javanica. Unimontes Científica, 6, 123-127.

Santos, C.A.F., da Costa, S.R., de Souza, R.R.C, Flori, J. E. \& Castro, J.M.C (2016). Compatibilidade de cruzamentos e aspectos genéticos para controle de Meloido gyne enterolobii (palestra 31). www.nematologia2016.com. $\mathrm{br} /$ files/Anais2016.zip

Sasser, J. N., Carter, C. C. \& Hartman, K. M. (1984). Standardization of host suitability studies and reporting of resistance to root knot nematodes. Crop Nematode Research \& Control Project (4p). ISBN 0-931901-03-0. Raleigh: North Carolina State University Graphics.

Sheela, M. S. (1995). Analysis of phytonematodes associated with coconut-based cropping system in Kerala. Indian Coconut Journal, 26, 5-6.

Siqueira, K. M. S., Freitas, V. M., Almeida, M. R. A., Santos, M. F. A., Cares, J. E., Tigano, M. S., \& Carneiro, R. M. D. G. (2009). Detecção de Meloidogyne mayaguensis em goiabeira e mamoeiro no estado de Goiás, usando marcadores moleculares. Tropical Plant Pathology, 34, 256-260.

Souza, R. M., Nogueira, M. S., Lima, I. M., Melarato, M., \& Dolinski, C. M. (2006). Management of the guava rootknot nematode in Sao Joao da Barra, Brazil, and report of new hosts. Nematologia Brasileira, 30, 165-169.

Tigano, M., Siqueira, K., Castagnone-Sereno, P., Mulet, K., Queiroz, P., Santos, M., Teixeira, C., Almeida, M., Silva, J., \& Carneiro, R. (2010). Genetic diversity of the root-knot nematode Meloidogyne enterolobii and development for SCAR marker for this guava-damaging species. Plant Pathology, 59, 1054-1061. 